\title{
ASSOCIATED POLYNOMIALS, SPECTRAL MATRICES AND THE BISPECTRAL PROBLEM
}

\author{
F. Alberto Grünbaum and Luc Haine \\ Dedicated to Professor Richard Askey on the occasion of his 65th birthday.
}

\begin{abstract}
The associated Hermite, Laguerre, Jacobi, and Bessel polynomials appear naturally when Bochner's problem [5] of characterizing orthogonal polynomials satisfying a second-order differential equation is extended to doubly infinite tridiagonal matrices. We obtain an explicit expression for the spectral matrix measure corresponding to the associated doubly infinite tridiagonal matrix in the Jacobi case. We show that, in an appropriate basis of "bispectral" functions, the spectral matrix can be put into a nice diagonal form, restoring the simplicity of the familiar orthogonality relations satisfied by the Jacobi polynomials.
\end{abstract}

\section{Introduction}

This paper deals with topics that are very classical. We did arrive at them, however, by a fairly nonclassical route: the study of the bispectral problem. We feel that this gives useful insight into subjects that have been considered earlier for different reasons. At any rate, we hope that Dick Askey, to whom this paper is dedicated, will like this interweaving of old and new material. His own mastery at doing this has proved tremendously useful to mathematics and to all of us.

We start with a statement of the bispectral problem which is appropriate for our purposes: describe all families of functions $f_{n}(z), n \in \mathbb{Z}, z \in \mathbb{C}$, that satisfy

$$
(L f)_{n}(z)=z f_{n}(z) \text { and } B f_{n}(z)=\lambda_{n} f_{n}(z) \text { for all } z \text { and } n \text {. }
$$

Here $L$ is an arbitrary doubly infinite tridiagonal matrix and $B$ is an arbitrary differential operator. The entries of $L$ depend solely on $n$, and the coefficients of $B$ solely on $z$.

When $B$ has order two and $L$ is assumed to have $L_{n, n+1}=1$ and $L_{n, n-1}$ nonzero, the complete answer, given in [11], see also [13], can. be restated as follows: pick numbers $a, b, c$, and pick any solution $w(z)$ of Gauss' hypergeometric equation

$$
B w=z(1-z) D^{2} w+(c-(a+b+1) z) D w-a b w=0 \text { with } D=d / d z .
$$

This determines a family $f_{n}(z)$, such that

$$
B f_{n}(z)=\lambda_{n} f_{n}(z), \quad f_{0}(z)=w(z),
$$

and

$$
(L f)_{n}(z)=z f_{n}(z)
$$

Received September 6, 1998, revised October 14, 1998.

1991 Mathematics Subject Classification: 33C45, 34L05.

Key words and phrases: orthogonal polynomials and functions, spectral theory. 
for an appropriate doubly infinite tridiagonal matrix $L$. Conversely, this is, in the generic case, the solution to the bispectral problem when $B$ has order two. The remaining cases, depending on fewer parameters, are connected with the names of Hermite, Laguerre, and Bessel, whereas the one above is related to Jacobi. They are all discussed in detail in [11] and [13].

Notice that one could choose $f_{0}(z)$ in an arbitrary way, for instance $f_{0}(z)=1$, as we did in [11]. This has the effect of conjugating $B$ by $f_{0}(z)$.

Two words about the $q$-world. In [12], we have formulated the corresponding bispectral question when $B$ is a second-order $q$-difference operator, and we have shown that what we call the Gauss-Askey-Wilson equation plays exactly the role that Gauss' equation plays in the case $q=1$.

The bispectral problem was introduced in the case when both $L$ and $B$ are differential operators in [9]. For further material on the bispectral problem, we refer to the Proceedings of a recent workshop at CRM, Montréal; see [14].

One of the most interesting issues behind the bispectral problem is that when $L$ is of the form

$$
-d^{2} / d x^{2}+V(x)
$$

and one allows for $B$ differential operators of arbitrary order, then the $V$ 's that result in bispectral situations naturally organize themselves into smooth manifolds on which nice nonlinear evolutions, such as the Korteweg-deVries (or more generally $K P$ when $L$ has higher order) hierarchy or their master symmetries (the Virasoro flows), act naturally.

A similar situation arises in the case when $L$ is a tridiagonal matrix, as has been discussed in [11]. In this case, the simplest of these evolutions corresponds to translation, i.e., shifting the index $n$ by $t$ (a real number). This requires that the entries of $L$ have nice (say rational) expressions in terms of $n$. The next simplest evolution is given by the Toda flow. This gives a connection with a more classical point of view. Indeed, for experts on orthogonal polynomials, the first of these flows should sound rather familiar: it gives rise to the notion of "associated polynomials."

The subject of associated orthogonal polynomials was first considered in the papers [19] and [6] and then in the paper of Askey and Wimp [3] where the spectral measure for Laguerre and Hermite (associated) polynomials is determined, and at about the same time in the article by Askey and Ismail [2] where some polynomials of Al-Salam and Chihara are considered. We are thankful for the first two references to Chihara; see [7]. The idea is very natural: consider those polynomials satisfying the standard recursion relation satisfied by these polynomials with $n$ replaced by $n+t$, for arbitrary real $t$. Some later papers, where this is extended to other cases, include the very influential paper by Wimp [21] where the Jacobi case is done, and then the papers by Ismail, et al. [16] where the Wilson polynomials are considered as well as that of Ismail and Rahman [17], which gives results for the Askey-Wilson polynomials.

It is one of the points made in this paper that the bispectral problem leads in a totally natural way to consider these objects. In fact, it was observed in [13] that all the solutions of the bispectral problem-given in [11] when $B$ has order twoare obtained by taking the tridiagonal matrices that correspond to either the Jacobi, Laguerre, Hermite, or Bessel polynomials and extending them to all integers, as well as introducing an extra parameter $t$ by allowing for the above mentioned translation. 
From our point of view, the "associated polynomials," or more precisely the doubly infinite (symmetric) tridiagonal matrices with $a_{n}(t)$ and $b_{n}(t)$ as given in Section 2 below, constitute the simplest possible "evolutions" of these objects away from their value at $t=0$. We will refer to these matrices as the "associated Jacobi matrices."

We consider these doubly infinite matrices as the simplest (generic) instances where the bispectral property holds, and conjecture - as in [11] - that all other solutions of the bispectral problem (for tridiagonal $L$ ) are obtained by applications of the Darboux process to properly chosen "associated Jacobi matrices." We will return to this point in a later publication.

Since these objects are likely to play such a basic role, we look into the corresponding "harmonic analysis" that they give rise to. There are different choices to be made, and we take up here the analog of the "sine and cosine transforms" on the integers.

Given "properly defined eigenfunctions" of $L$, denoted here by $p_{n}(z)$ and $q_{n}(z)$, (see (3.2), (3.3) for details), one introduces a pair of "transforms" which map sequences $c_{n}$ in $L^{2}(\mathbb{Z})$ to functions

$$
F(z)=\sum_{-\infty}^{\infty} c_{n} p_{n}(z) \text { and } G(z)=\sum_{-\infty}^{\infty} c_{n} q_{n}(z) .
$$

The issue of inverting these transforms amounts to finding a "spectral matrix"

$$
\sigma(z)=\left(\begin{array}{ll}
\sigma_{11}(z) & \sigma_{12}(z) \\
\sigma_{21}(z) & \sigma_{22}(z)
\end{array}\right)
$$

such that the coefficients $c_{n}$ above are given by

$$
\begin{array}{rl}
c_{n}=\int_{\mathbb{R}} & F(z) p_{n}(z) \sigma_{22}(z) d z+\int_{\mathbb{R}} F(z) q_{n}(z) \sigma_{21}(z) d z \\
& \quad+\int_{\mathbb{R}} G(z) p_{n}(z) \sigma_{12}(z) d z+\int_{\mathbb{R}} G(z) q_{n}(z) \sigma_{11}(z) d z .
\end{array}
$$

If one introduces the convenient notation, see [4],

$$
P_{n}(z)=\left(\begin{array}{cc}
q_{-n-1}(z) & p_{-n-1}(z) \\
q_{n}(z) & p_{n}(z)
\end{array}\right)
$$

for $n$ non-negative, one can express the condition above as

$$
\int_{\mathbb{R}} P_{n}(z) \sigma(z) P_{m}^{T}(z) d z=\delta_{n m} I, \quad n, m \text { non-negative. }
$$

We now can state the main result in this paper. We obtain an explicit expression for the spectral (matrix) measure corresponding to the associated Jacobi matrices. The spectral matrix described above can (in principle) be determined for any doubly infinite symmetric tridiagonal matrix by using the method that goes back to Weyl, Titchmarsh, and Kodaira or (if one so chooses) the notion of numerator and denominator polynomials as in Stieltjes. These numerator polynomials give, incidentally, the simplest and earliest instances of associated or shifted polynomials, corresponding to $t=1$. The cases where this program can be implemented explicitly are indeed very few. For instance, the paper [18], which deals with some of these topics, stops short of doing this. See their remark at the end of Section 3. Here we accomplish this by exploiting (rather implicitly) the bispectral property of this situation.

In carrying out this program, we have found it very convenient to use some of the tools that were developed in the remarkable paper by Wimp [21]. The spectral matrix 
that we find involves functions that are just as complicated as the ones that enter into Wimp's expression for the weight function of the associated Jacobi polynomials.

From our perspective, doubly infinite matrices are more basic than the semi-infinite ones that appear in the theory of orthogonal polynomials. They permit one to deal, for instance, with the case when no boundary condition at $n=-1$ is appropriate, and they have a two-dimensional space of eigenfunctions which allows one to perform bispectral Darboux transformations. In this light, we find it rather pleasing that, by an appropriate change of basis, we can convert the spectral matrix into a nice diagonal form involving exactly the time-honored weight function of the Jacobi polynomials themselves, namely

$$
\rho(z)=z^{c-1}(1-z)^{a+b-c} .
$$

This can be attributed to bispectrality, since Gauss' hypergeometric operator

$$
B=z(1-z) D^{2}+(c-(a+b+1) z) D
$$

can be written as

$$
B=1 / \rho D p D \text { with } p=\rho z(1-z) .
$$

It is then natural to consider this operator in $L^{2}(\rho)$.

Although our proof of the orthogonality relations (4.12) relies on the general spectral theory of self-adjoint second-order difference operators, it is possible to exploit more directly the fact that the functions $\tilde{u}_{n}, \tilde{v}_{n}$ that enter in (4.12) are eigenfunctions of $B$, as well as of $L$. That is, exploiting bispectrality, one can show rather easily that for $n$ different from $m$, the integral is zero. This is done by the usual Wronskian computation under the conditions $0<c<2$ and $-1<c-a-b<1$, which incidentally make $B$ "limit circle" at both endpoints; see Remark 2 at the end of Section 4.

We have strong reasons to believe that in the cases studied here, namely $0<c<2$ and $-1<c-a-b<1, L$ has no discrete spectrum. That is, we have found its complete spectral resolution.

\section{The bispectral route to the associated Jacobi matrix}

In this section, we summarize the results of [11] where the complete solution of the discrete-continuous version of the bispectral problem (1.1) (with $B$ of order 2) was obtained. We shall restrict ourselves to the generic solution of the problem which will concern us in this paper. It is described in terms of Gauss' hypergeometric equation:

$$
z(1-z) w^{\prime \prime}(z)+(c-(a+b+1) z) w^{\prime}(z)-a b w(z)=0
$$

for an (almost) arbitrary choice of its three parameters $a, b$, and $c$.

Let

$$
a_{n}=\frac{(n+a-1)(n-b)(n-c+a)(n+c-b-1)}{(2 n-b+a-2)(2 n-b+a-1)^{2}(2 n-b+a)}
$$

and

$$
b_{n}=\frac{1}{2}\left(\frac{(b+a-1)(2 c-b-a-1)}{(2 n-b+a-3)(2 n-b+a-1)}+1\right) .
$$

From the point of view of the bispectral problem, we need to assume that $a_{n} \neq 0$ for $n=0,-1,-2, \ldots$, and that $a, b$ are such that (2.2) and (2.3) make sense for all $n$. See [11]. We will assume here that $a_{n}$ is positive for all $n$. This restriction can be removed in certain cases, but we do not pursue this here. 
We pick $w(z)$ as an arbitrary solution of Gauss' equation and define $f_{0}(z)$ and $f_{1}(z)$ by

$$
f_{0}(z)=w(z)
$$

and

$$
f_{1}(z)=\frac{1}{(b-a) \sqrt{a_{1}}}\left(z(1-z) w^{\prime}(z)-a\left(z+\frac{b-c}{a-b+1}\right) w(z)\right) .
$$

We define $f_{n}(z)$ for $n \in \mathbb{Z}, n \neq 0,1$, via the three-term recursion relation

$$
z f_{n}(z)=\sqrt{a_{n}} f_{n-1}(z)+b_{n+1} f_{n}(z)+\sqrt{a_{n+1}} f_{n+1}(z) .
$$

It is shown in [11] that the functions $f_{n}(z)$ are eigenfunctions of a second-order differential operator

$$
B f_{n}(z)=\lambda_{n} f_{n}(z)
$$

with

$$
\lambda_{n}=(a+n)(b-n) \text { and } B=z(1-z) D^{2}+(c-(a+b+1) z) D, \quad D=\frac{d}{d z},
$$

and that (generically) when (2.6) and (2.7) hold simultaneously, we have this case.

We observe that if we put

$$
c=\beta+1, \quad b=-t, \quad a=\alpha+\beta+1+t,
$$

in (2.2) and (2.3), we can write

$$
a_{n}=\tilde{a}_{n+t}, \quad b_{n}=\tilde{b}_{n+t},
$$

with $\tilde{a}_{n}$ and $\tilde{b}_{n}$ the coefficients of the standard three-term recursion relation satisfied by the monic Jacobi polynomials shifted to the interval $[0,1]$ :

$$
\begin{aligned}
\tilde{a}_{n} & =\frac{n(n+\alpha)(n+\beta)(n+\alpha+\beta)}{(2 n+\alpha+\beta-1)(2 n+\alpha+\beta)^{2}(2 n+\alpha+\beta+1)}, \\
\tilde{b}_{n} & =\frac{1}{2}\left(\frac{\beta^{2}-\alpha^{2}}{(2 n+\alpha+\beta-2)(2 n+\alpha+\beta)}+1\right) .
\end{aligned}
$$

This simple observation connects the solution of the discrete-continuous version of the bispectral problem (for an operator $B$ of order 2) to the concept of the associated polynomials, which is familiar to experts in the theory of orthogonal polynomials. For this reason, we propose to call the doubly infinite (symmetric) tridiagonal matrix defining the three-term recursion relation (2.6), with $a_{n}$ and $b_{n}$ as in (2.2) and (2.3), the associated Jacobi matrix.

Here we make some comments on the restrictions on $a, b, c$ that insure that all $a_{n}$ are positive. This condition also will ensure that two expressions involving $a, b, c-$ see (4.8) - are positive. We now see that this implies the pair of conditions $0<c<2$ and $-1<c-a-b<1$ (equivalent to $-1<\alpha<1$ and $-1<\beta<1$ ) whose use will be mentioned below.

From (2.10) and (2.11), the expression for $a_{n}$ is

$$
\frac{(t+n)(t+n+\alpha)(t+n+\beta)(t+n+\beta+\alpha)}{(2 t+2 n+\beta+\alpha-1)(2 t+2 n+\beta+\alpha)^{2}(2 t+2 n+\beta+\alpha+1)} .
$$

We consider $\alpha, \beta$ as given, and we aim at finding conditions that ensure that some $t$ can be found to make this expression well-defined and positive for all $n$. 
It is clear that $t$ should be chosen so that the product

$$
\begin{aligned}
(t+n)(t+n+\alpha)(t+n+\beta)(t+n & +\beta+\alpha) \\
& \times(2 t+2 n+\beta+\alpha-1)(2 t+2 n+\beta+\alpha+1)
\end{aligned}
$$

is positive for each $n$. This means that, for each fixed $n$, one considers the six values (in some appropriate order)

$$
-n-\frac{\beta}{2}-\frac{\alpha}{2}-\frac{1}{2}, \quad-n-\frac{\beta}{2}-\frac{\alpha}{2}+\frac{1}{2}, \quad-n-\beta-\alpha, \quad-n-\beta, \quad-n-\alpha, \quad-n
$$

and the five consecutive intervals that they determine. Then one requires that $t$ should lie either outside all of them or in the second or fourth of these intervals. As $n$ changes, these six points just keep shifting by $n$, and the existence of a $t$ that meets these requirements is an issue of determining the common intersection of this sliding set of five consecutive intervals.

Things are particularly nice when the smallest value among these above is the first one in the list, and the largest is the second one. In this case, they fill out an interval of length one, and the discussion of intersections for different values of $n$ becomes trivial. For instance, if $\beta>\alpha>0$, we can choose $t$ in the union of the intervals

$$
[-(\alpha+\beta)+1,-\beta+1] \text { and }[-\alpha+1,1] \text {. }
$$

This "nice" case is equivalent to the conditions

$$
-1<\alpha+\beta<1 \text { and }-1<\alpha-\beta<1 .
$$

These conditions cut out a nice diamond in the square

$$
-1<\alpha<1, \quad-1<\beta<1 \text {. }
$$

With a little bit of extra work, one can see that, for any point in this square, some $t$ can be chosen in such a way that positivity is ensured. It is not hard to see that as soon as we are out of this square, there is no value of $t$ that makes all $a_{n}$ positive.

The associated Jacobi polynomials $p_{n}(z ; t), n=0,1,2, \ldots$, are defined to be the solution of the three-term recursion relation (2.6), with $a_{n}, b_{n}$ as in (2.10), (2.11) and

$$
p_{-1}(z ; t)=0, \quad p_{0}(z ; t)=1 \text {. }
$$

For $t=0$, these polynomials coincide with the classical Jacobi polynomials (appropriately normalized) and shifted to the interval $[0,1]$. As mentioned in the introduction, the associated Jacobi polynomials were studied extensively in a beautiful paper by Wimp [21], of which we were unaware at the time of writing [11]. As soon as $t \neq 0$, these polynomials are not eigenfunctions of a second-order differential operator. (Otherwise, they would be classical!) They are however, as Wimp observed, solutions of a fourth-order differential equation with $n$ dependent coefficients that are polynomials in $z$. This equation was determined in [21] by the use of the computer algebra package Macsyma. Equations of this type, with order as low as two, hold for orthogonal polynomials with fairly general weight functions; see [8] and its references. The coefficients are determined in terms of the weight function, and this is practical only in some cases.

As found in [11], any family of bispectral functions can be obtained by solving the recursion relation (2.6) with initial conditions $f_{0}(z)$ and $f_{1}(z)$ given by (2.4) and (2.5). In this paper, we shall use two different bases of bispectral functions, which we now describe. 
A first basis $\left\{g_{n}(z), h_{n}(z)\right\}$ of bispectral functions is obtained by changing $n$ into $n+t$ in the classical formulas expressing the Jacobi polynomials $P_{n}^{(\alpha, \beta)}$ and the Jacobi functions of the second kind $Q_{n}^{(\alpha, \beta)}$, shifted to the interval [0,1], and appropriately normalized to satisfy the symmetric version (2.6) of the three-term recursion relation. We define inductively $k_{n}, n \in \mathbb{Z}$, via

$$
k_{0}=1, \quad k_{n}=k_{n-1} / \sqrt{a_{n}} .
$$

Using the classical formulas in terms of Gauss' hypergeometric function ${ }_{2} F_{1}(a, b ; c ; z)$, for the Jacobi polynomials and the Jacobi functions of the second kind ([10], vol. 2, p. $170,(16)$ and (18)), remembering (2.9), we find that

$$
\begin{aligned}
g_{n}(z)= & k_{n} \frac{(t+1)_{n}(\alpha+\beta+1+t)_{n}}{(\alpha+\beta+1+2 t)_{2 n}} P_{n+t}^{(\alpha, \beta)}(2 z-1) \\
= & k_{n} \frac{\Gamma(a-c+1+n)(a)_{n}}{\Gamma(a+b-c+1) \Gamma(1-b)(a-b)_{2 n}} \\
& \quad \times{ }_{2} F_{1}(a+n, b-n ; a+b-c+1 ; 1-z), \quad|z-1|<1,
\end{aligned}
$$

and

$$
\begin{aligned}
h_{n}(z)=k_{n} & \frac{(t+1)_{n}(\alpha+\beta+1+t)_{n}}{(\alpha+\beta+1+2 t)_{2 n}} Q_{n+t}^{(\alpha, \beta)}(2 z-1) \\
=k_{n} & \frac{(a)_{n}(1-b)_{n}}{(a-b)_{2 n}} \\
& \times \frac{\Gamma(a-c+1+n) \Gamma(c-b+n)}{2 \Gamma(a-b+1+2 n)} z^{1-c}(z-1)^{c-a-1-n} \\
& \quad \times{ }_{2} F_{1}\left(a-c+1+n, 1-b+n ; a-b+1+2 n ; \frac{1}{1-z}\right), \quad|z-1|>1 .
\end{aligned}
$$

Here, as well as in the rest of the paper, $(a)_{n}=\Gamma(a+n) / \Gamma(a)$ denotes the standard Pochhammer's symbol. In the next section, we shall need appropriate analytic extensions of these formulas; see (3.25), (3.26).

The second basis $\left\{u_{n}(z), v_{n}(z)\right\}$ of bispectral functions that we shall need corresponds to the standard choice for a basis of solutions of the hypergeometric equation around $z=0$ :

$$
u_{0}(z)={ }_{2} F_{1}(a, b ; c ; z) \text { and } v_{0}(z)=z^{1-c}{ }_{2} F_{1}(a-c+1, b-c+1 ; 2-c ; z) .
$$

It can be shown that the solutions of the three-term recursion relation (2.6), with $u_{0}(z)$ and $v_{0}(z)$ as in (2.16) and $u_{1}(z)$ and $v_{1}(z)$ defined in the manner of (2.5), are given by

$$
\begin{aligned}
& u_{n}(z)=k_{n} \alpha_{n} F_{1}(a+n, b-n ; c ; z), \\
& v_{n}(z)=k_{n} \beta_{n} z^{1-c}{ }_{2} F_{1}(a-c+1+n, b-c+1-n ; 2-c ; z)
\end{aligned}
$$

with

$$
\begin{aligned}
& \alpha_{n}=(-1)^{n} \frac{(a)_{n}(c-b)_{n}}{(a-b)_{2 n}} \\
& \beta_{n}=(-1)^{n} \frac{(a+1-c)_{n}(1-b)_{n}}{(a-b)_{2 n}}
\end{aligned}
$$




\section{The spectral matrix}

The spectral theory of second-order difference operators

$$
(L f)_{n}=\sqrt{a_{n}} f_{n-1}+b_{n+1} f_{n}+\sqrt{a_{n+1}} f_{n+1}, \quad a_{n}>0 \text { and } b_{n} \in \mathbb{R},
$$

acting in the Hilbert space $L^{2}(\mathbb{Z})$ of sequences $f=\left(\ldots, f_{-1}, f_{0}, f_{1}, \ldots\right)^{T}$ such that $\sum_{n=-\infty}^{\infty}\left|f_{n}\right|^{2}<\infty$, mimics the classical Weyl-Titchmarsh-Kodaira theory for secondorder differential operators on the line. A nice exposition of this theory can be found in Berezanskii [4], Chapter 7. We thank H. Flaschka for this useful reference. There may be other treatments, but we are not aware of them. This is entirely analogous to the way in which the treatment of differential operators in $L^{2}(0, \infty)$ due to Weyl [20] was adapted for the treatment of orthogonal polynomials by Hellinger [15]. A nice account of this material is in Akhiezer's book [1]. The case of doubly infinite matrices (operators on the whole axis) that concerns us in this paper differs from the case of semi-infinite matrices (operators on the semi-axis) in that now, in general, the spectrum will have multiplicity two. This multiplicity of the spectrum leads, instead of to a spectral measure, to a two-dimensional matrix measure. In the so-called limit point case, the spectral matrix is unique corresponding to the fact that the symmetric operator $L$ has only one self-adjoint extension. In this section, after a brief summary of the general theory which closely follows [4], we compute explicitly the spectral matrix for the associated Jacobi matrix $L$ as defined by (2.2) and (2.3).

Let us denote by $p_{n}(z)$ and $q_{n}(z), n \in \mathbb{Z}, z \in \mathbb{C}$, the polynomial solutions of $L f=z f$, with initial conditions

$$
p_{-1}=0, \quad p_{0}=1
$$

and

$$
q_{-1}=1, \quad q_{0}=0 .
$$

Introduce then, for $n=0,1,2, \ldots$, the following $2 \times 2$ matrices

$$
P_{n}=\left(\begin{array}{cc}
q_{-n-1} & p_{-n-1} \\
q_{n} & p_{n}
\end{array}\right)
$$

and

$$
Q_{n}=-\frac{1}{\sqrt{a_{0}}}\left(\begin{array}{cc}
p_{-n-1} & 0 \\
0 & q_{n}
\end{array}\right)
$$

Let $L^{2}\left(\mathbb{Z}_{+}\right)$be the Hilbert space of sequences of complex numbers $f=\left(f_{0}, f_{1}, f_{2}, \ldots\right)^{T}$, such that $\sum_{n=0}^{\infty}\left|f_{n}\right|^{2}<\infty$. The so-called limit point case is characterized by the existence of a unique $2 \times 2$ matrix $M(z)$, for $z \in \mathbb{C}$ with $\operatorname{Im} z>0$, such that for each $i, j \in\{1,2\}$ the sequence

$$
\left(Q_{n}(z)+P_{n}(z) M(z)\right)_{i j} \in L^{2}\left(\mathbb{Z}_{+}\right) .
$$

We will see below that this is the case for the associated Jacobi matrix. In this case, one can compute $M(z)$ by adapting the way in which Stieltjes studied continued fractions arising from a (one-sided) three-term recursion. This was, in fact, one of the ways in which orthogonal polynomials first arose, in terms of "numerator and denominator polynomials"; see [1]. Thus, we propose to compute $M(z)$ as the limit

$$
M(z)=-\lim _{n \rightarrow \infty} P_{n}^{-1}(z) Q_{n}(z) .
$$


The matrix $M(z)$ is the Stieltjes transform of the spectral matrix $d \Sigma(x)$ :

$$
M(z)=\int_{-\infty}^{\infty} \frac{1}{x-z} d \Sigma(x), \quad \operatorname{Im} z>0 .
$$

Equivalently, $d \Sigma(x)$ is obtained via the Stieltjes inversion formula

$$
\frac{\Sigma(x+0)+\Sigma(x-0)}{2}-\frac{\Sigma(c+0)+\Sigma(c-0)}{2}=\frac{1}{\pi} \lim _{\substack{v \rightarrow 0 \\ v>0}} \int_{c}^{x} \operatorname{Im} M(u+i v) d u .
$$

Moreover, the following orthogonality relations hold

$$
\int_{-\infty}^{\infty} P_{m}(x) d \Sigma(x) P_{n}(x)^{T}=\delta_{m n} I
$$

with $I$ denoting the $2 \times 2$ identity matrix.

From (3.4), (3.5), and (3.7), one computes easily that

$$
M(z)=\frac{1}{\sqrt{a_{0}}}\left(\begin{array}{ll}
\frac{1}{m_{2}(z)-m_{1}(z)} & \frac{m_{1}(z)}{m_{1}(z)-m_{2}(z)} \\
\frac{m_{1}(z)}{m_{1}(z)-m_{2}(z)} & \frac{m_{1}(z) m_{2}(z)}{m_{2}(z)-m_{1}(z)}
\end{array}\right)
$$

with

$$
m_{1}(z)=\lim _{n \rightarrow+\infty} \frac{q_{n}(z)}{p_{n}(z)}
$$

and

$$
m_{2}(z)=\lim _{n \rightarrow+\infty} \frac{q_{-n-1}(z)}{p_{-n-1}(z)}
$$

In the case of the associated Jacobi matrix defined in (2.2) and (2.3), we observe that since

$$
\lim _{n \rightarrow \pm \infty} \sqrt{a_{n}}=\frac{1}{4} \text { and } \lim _{n \rightarrow \pm \infty} b_{n}=\frac{1}{2}
$$

the operator $L$ in (3.1) is bounded; thus, $L$ is self-adjoint on $L^{2}(\mathbb{Z})$, which puts us automatically in the limit point case, for which the spectral matrix is unique. The rest of the section is devoted to the explicit computation of the spectral matrix. As said in the introduction, the cases where this can be done are very few. In fact, the only explicit example that we could find is in [4], which discusses the case where $\sqrt{a_{n}}=1 / 4$ and $b_{n}=1 / 2$, for all $n \in \mathbb{Z}$. Notice that this corresponds precisely to the asymptotic behavior (3.14) of our family and indeed, this case is obtained for a very special choice of the parameters $a, b$, and $c$; for instance, one can pick $c=3 / 2$ and $b=1-a$, with an arbitrary value for the parameter $a$.

In what follows, all the quantities that we shall compute depend on the three parameters $a, b$, and $c$ or, equivalently, the three parameters $\alpha, \beta$, and $t$, as defined in (2.9). Whenever needed, we shall indicate this dependence; for instance, we shall write $a_{n}(a, b, c)$ or $a_{n}(t)$ if we want to stress the dependence of $a_{n}$ on $(a, b, c)$ or on $t$. The following observation, which follows from the definition of $a_{n}$ and $b_{n}$ in (2.2) and (2.3), will simplify our task

$$
a_{-n}(a, b, c)=a_{n}(b+1, a-1, c) \quad \text { and } \quad b_{-n}(a, b, c)=b_{n+1}(b+1, a-1, c) .
$$


Using the definition of the polynomials $p_{n}(z ; a, b, c)$ and $q_{n}(z ; a, b, c)$ in (3.2) and (3.3), it implies that

$$
\begin{aligned}
& q_{-n}(z ; a, b, c)=p_{n-1}(z ; b+1, a-1, c), \\
& p_{-n}(z ; a, b, c)=q_{n-1}(z ; b+1, a-1, c),
\end{aligned}
$$

and therefore, by (3.12) and (3.13), we get that

$$
m_{2}(z ; a, b, c)=\frac{1}{m_{1}(z ; b+1, a-1, c)},
$$

which cuts our labor in half. We now determine $m_{1}(z ; a, b, c)$. Our method is inspired by Wimp's computation [21] of the spectral measure for the associated Jacobi polynomials.

The polynomials $p_{n}(z ; t)$ in (3.2) (only the dependence in $t$ will concern us in what follows) can be expressed in terms of the basis $\left\{g_{n}(z ; t), h_{n}(z ; t)\right\}$ introduced in (2.14) and (2.15):

$$
p_{n}(z ; t)=\frac{h_{-1}(z ; t) g_{n}(z ; t)-g_{-1}(z ; t) h_{n}(z ; t)}{h_{-1}(z ; t) g_{0}(z ; t)-g_{-1}(z ; t) h_{0}(z ; t)} .
$$

Also, it follows from the definition of $p_{n}(z ; t)$ and $q_{n}(z ; t)$ in (3.2) and (3.3) that

$$
q_{n}(z ; t)=-\sqrt{\frac{a_{0}(t)}{a_{1}(t)}} p_{n-1}(z ; t+1) .
$$

By the very definition of the functions $g_{n}$ and $h_{n}$ in (2.14) and (2.15), which are obtained by shifting $n$ to $n+t$ in the classical formulas defining the Jacobi polynomials and the Jacobi functions of the second kind, we have that

$$
g_{n}(z ; t+1)=\delta g_{n+1}(z ; t) \text { and } h_{n}(z ; t+1)=\delta h_{n+1}(z ; t),
$$

with $\delta$ a constant independent on $n$.

Combining (3.18), (3.19), and (3.20), we obtain that

$$
\begin{aligned}
\frac{q_{n}(z ; t)}{p_{n}(z ; t)}=-\sqrt{\frac{a_{0}(t)}{a_{1}(t)}} \frac{h_{-1}(z ; t) g_{0}(z ; t)-g_{-1}(z ; t) h_{0}(z ; t)}{h_{0}(z ; t) g_{1}(z ; t)-g_{0}(z ; t) h_{1}(z ; t)} \\
\times \frac{h_{0}(z ; t) g_{n}(z ; t)-g_{0}(z ; t) h_{n}(z ; t)}{h_{-1}(z ; t) g_{n}(z ; t)-g_{-1}(z ; t) h_{n}(z ; t)} \\
=-\frac{h_{0}(z ; t) g_{n}(z ; t)-g_{0}(z ; t) h_{n}(z ; t)}{h_{-1}(z ; t) g_{n}(z ; t)-g_{-1}(z ; t) h_{n}(z ; t)}
\end{aligned}
$$

The last equality follows from the standard "Casorati type" formulas expressing the entries of the operator $L-z I$, in terms of its two-dimensional kernel $\left\{g_{n}(z), h_{n}(z)\right\}$, which gives in particular that

$$
\frac{\sqrt{a_{n}}}{\sqrt{a_{n+1}}}=\frac{g_{n}(z) h_{n+1}(z)-g_{n+1}(z) h_{n}(z)}{g_{n-1}(z) h_{n}(z)-g_{n}(z) h_{n-1}(z)} .
$$

Now, as observed in [21], $g_{n}$ dominates $h_{n}$ exponentially in $\mathbb{C} \backslash[0,1]$

$$
\begin{gathered}
\frac{h_{n}(z)}{g_{n}(z)} \sim C(z) \tau(z)^{-4 n}\left(1+\frac{c_{1}}{n}+\frac{c_{2}}{n^{2}}+\ldots\right), \\
\tau(z)=\sqrt{z}+\sqrt{z-1}, \quad n \rightarrow+\infty, \quad z \in \mathbb{C} \backslash[0,1],
\end{gathered}
$$


and thus, from the definition of $m_{1}(z)$ in (3.12), using (3.21), we deduce that

$$
m_{1}(z)=-\frac{h_{0}(z)}{h_{-1}(z)}, \quad z \in \mathbb{C} \backslash[0,1] .
$$

The formula for $h_{n}(z)$ given in (2.15) is valid for $|z-1|>1$. Using a Kummer transformation ([10], vol. 1, p. 105, (4)) produces a formula which is valid for $|z|>1$ :

$$
\begin{array}{r}
h_{n}(z)=k_{n} \frac{(a)_{n}(1-b)_{n} \Gamma(a-c+1+n) \Gamma(c-b+n)}{2(a-b)_{2 n} \Gamma(a-b+1+2 n)} z^{b-c-n}(z-1)^{c-a-b} \\
\quad \times{ }_{2} F_{1}\left(c-b+n, 1-b+n ; a-b+1+2 n ; \frac{1}{z}\right),
\end{array}
$$

$|z|>1$. From (2.15), (3.11), (3.17), (3.24), and (3.25), we see that, as long as $x \notin[0,1]$, the imaginary part $\operatorname{Im} M(x)=0$, and thus, by (3.9), the spectral matrix vanishes outside of the interval $[0,1]$. Here we assume implicitly that there are no discrete point masses. (We have strong evidence that this is the case; see Remark 2 at the end of Section 4.) In order to compute the spectral matrix, we need to find the analytic continuation of $h_{n}(z)$ when $z$ tends to the cut $[0,1]$ from above the real axis. Using the analytic continuation formula ([10], vol. 1, p. 108, (2)), we get

$$
\begin{gathered}
h_{n}(z)=\frac{k_{n} e^{i \pi(b-n-c)}}{2(a-b)_{2 n}}(z-1)^{c-a-b} \\
\quad \times\left\{\frac{\Gamma(1-c) \Gamma(c-b+n)(a)_{n}}{\Gamma(1-b)}{ }_{2} F_{1}(c-a-n, c-b+n ; c ; z)\right. \\
\quad+\frac{\Gamma(c-1) \Gamma(a-c+1+n)(1-b)_{n}}{\Gamma(a)}\left(e^{-i \pi} z\right)^{1-c} \\
\left.\times{ }_{2} F_{1}(1-a-n, 1-b+n ; 2-c ; z)\right\},
\end{gathered}
$$

$|z|<1,|\arg (-z)|<\pi$.

In what follows, we use the shorthand notation:

$$
\begin{array}{ll}
F_{1}={ }_{2} F_{1}(c-a, c-b ; c ; z), & F_{2}={ }_{2} F_{1}(1-a, 1-b ; 2-c ; z), \\
F_{3}={ }_{2} F_{1}(c-a+1, c-b-1 ; c ; z), & F_{4}={ }_{2} F_{1}(2-a,-b ; 2-c ; z) .
\end{array}
$$

From (3.24) and (3.26), we find, by a purely algebraic computation which uses some of the standard functional equations satisfied by the gamma function, that

$$
m_{1}(z ; a, b, c)=\gamma \frac{F_{1}+K\left(e^{-i \pi} z\right)^{1-c} F_{2}}{F_{3}+\nu K\left(e^{-i \pi} z\right)^{1-c} F_{4}},
$$

with

$$
\begin{gathered}
K=\frac{\Gamma(1-a) \Gamma(1-b) \Gamma(c-1) \sin (\pi a)}{\Gamma(c-a) \Gamma(c-b) \Gamma(1-c) \sin \pi(c-a)} \\
\nu=\frac{(a-1)(c-b-1)}{b(c-a)}
\end{gathered}
$$

and

$$
\gamma=\frac{(a-b)_{-2}}{\sqrt{a_{0}}(a)_{-1}(c-b)_{-1}}=\frac{\sqrt{a_{0}}(1+b)_{-1}(c-a+1)_{-1}}{(b-a+2)_{-2}}
$$


Remembering (3.17), we deduce easily from (3.27)-(3.31) that

$$
m_{2}(z ; a, b, c)=\gamma \frac{F_{1}+\mu K\left(e^{-i \pi} z\right)^{1-c} F_{2}}{F_{3}+\mu \nu K\left(e^{-i \pi} z\right)^{1-c} F_{4}}
$$

with

$$
\mu=\frac{\sin (\pi b) \sin \pi(c-a)}{\sin (\pi a) \sin \pi(c-b)} .
$$

Substituting (3.28) and (3.32) into (3.11) gives us explicit formulas for the entries $M_{i j}$ of the matrix $M(z)$, with $M_{12}=M_{21}$ :

$$
\begin{aligned}
& M_{11}=\frac{\left(e^{-i \pi} z\right)^{c-1}\left(F_{3}+\nu K\left(e^{-i \pi} z\right)^{1-c} F_{4}\right)\left(F_{3}+\mu \nu K\left(e^{-i \pi} z\right)^{1-c} F_{4}\right)}{\sqrt{a_{0}} \gamma(1-\mu) K\left(\nu F_{1} F_{4}-F_{2} F_{3}\right)}, \\
& M_{12}=\frac{\left(e^{-i \pi} z\right)^{c-1}\left(F_{1}+K\left(e^{-i \pi} z\right)^{1-c} F_{2}\right)\left(F_{3}+\mu \nu K\left(e^{-i \pi} z\right)^{1-c} F_{4}\right)}{\sqrt{a_{0}}(\mu-1) K\left(\nu F_{1} F_{4}-F_{2} F_{3}\right)} \\
& M_{22}=\frac{\gamma\left(e^{-i \pi} z\right)^{c-1}\left(F_{1}+K\left(e^{-i \pi} z\right)^{1-c} F_{2}\right)\left(F_{1}+\mu K\left(e^{-i \pi} z\right)^{1-c} F_{2}\right)}{\sqrt{a_{0}}(1-\mu) K\left(\nu F_{1} F_{4}-F_{2} F_{3}\right)}
\end{aligned}
$$

$|z|<1,|\arg (-z)|<\pi$.

Using the formula

$$
\begin{aligned}
{ }_{2} F_{1}(a+1, b-1 ; c ; z)= & \frac{1}{a(c-b)}\left\{z(1-z)(a-b+1) \frac{d}{d z}{ }_{2} F_{1}(a, b ; c ; z)\right. \\
& \left.+a(z(b-a-1)+(c-b)){ }_{2} F_{1}(a, b ; c ; z)\right\},
\end{aligned}
$$

we can express $F_{3}$ and $F_{4}$ in (3.27) in terms of $F_{1}$ and $F_{2}$, and we obtain

$$
\nu F_{1} F_{4}-F_{2} F_{3}=\frac{(a-b-1)(z-1) z^{c}}{b(c-a)} \times \operatorname{Wronskian}\left(F_{1}, z^{1-c} F_{2}\right) .
$$

Since $F_{1}$ and $z^{1-c} F_{2}$ are (independent) solutions of the same hypergeometric equation, the Wronskian in the last equation can easily be evaluated, which gives

$$
\nu F_{1} F_{4}-F_{2} F_{3}=\frac{(a-b-1)(1-c)(1-z)^{a+b-c}}{b(a-c)} .
$$

We are now in position to compute the spectral matrix. We already noticed that (under the assumption that there are no discrete point masses) it vanishes outside of $[0,1]$. Thus, by (3.9), we need to compute

$$
d \Sigma(x)=\Sigma^{\prime}(x) d x=\frac{1}{\pi} \lim _{\substack{y \rightarrow 0 \\ y>0}} \operatorname{Im} M(x+i y) d x, x \in[0,1],
$$

with $M(z)$ as in (3.34).

Using Kummer's relation ([10], vol. 1, p. $105,(2))$, we can rewrite the $F_{j}$ 's in (3.27) as

$$
F_{j}(x)=(1-x)^{a+b-c} G_{j}(x), \quad 1 \leq j \leq 4,
$$

with

$$
\begin{array}{ll}
G_{1}={ }_{2} F_{1}(a, b ; c ; x), & G_{2}={ }_{2} F_{1}(a-c+1, b-c+1 ; 2-c ; x), \\
G_{3}={ }_{2} F_{1}(a-1, b+1 ; c ; x), & G_{4}={ }_{2} F_{1}(a-c, b-c+2 ; 2-c ; x) .
\end{array}
$$


From (3.34), (3.37), (3.38), and (3.39), we obtain by a straightforward computation our main result:

Theorem 1. The spectral matrix of the associated Jacobi matrix is given by

$$
d \Sigma(x)= \begin{cases}0, & \text { for } x \notin[0,1], \\ \Sigma^{\prime}(x) d x, & \text { for } x \in[0,1],\end{cases}
$$

with the entries $\Sigma_{i j}^{\prime}, \Sigma_{12}^{\prime}=\Sigma_{21}^{\prime}$, of $\Sigma^{\prime}(x)$ given by

$$
\begin{aligned}
& \Sigma_{11}^{\prime}=\frac{L}{\gamma}\left(G_{3}^{2}-\nu^{2} \mu K^{2} x^{2(1-c)} G_{4}^{2}\right) \rho(x), \\
& \Sigma_{12}^{\prime}=-L\left(G_{1} G_{3}-\nu \mu K^{2} x^{2(1-c)} G_{2} G_{4}\right) \rho(x), \\
& \Sigma_{22}^{\prime}=\gamma L\left(G_{1}^{2}-\mu K^{2} x^{2(1-c)} G_{2}^{2}\right) \rho(x)
\end{aligned}
$$

where

$$
\begin{gathered}
\rho(x)=x^{c-1}(1-x)^{a+b-c}, \\
L=\frac{b(a-c) \sin \pi(1-c)}{\pi \sqrt{a_{0}}(a-b-1)(1-c)(1-\mu) K},
\end{gathered}
$$

and $K, \nu, \gamma, \mu, G_{1}, G_{2}, G_{3}, G_{4}$ are defined as in (3.29)-(3.31), (3.33), and (3.40).

\section{The diagonal form}

As recalled in Section 2, the associated Jacobi matrix arises naturally in the context of the bispectral problem; it is the generic doubly infinite tridiagonal matrix for which some family of eigenfunctions is also a family of eigenfunctions of a second-order differential operator. In fact, the vector space of common eigenfunctions is two-dimensional. The most natural choice for this space is provided by picking the standard basis of solutions for the hypergeometric equation around $z=0$, which leads to the functions $\left\{u_{n}(z)\right\}$ and $\left\{v_{n}(z)\right\}$ in (2.17), which are defined for $|z|<1$. In this section, we show that, in this basis, the spectral matrix becomes diagonal, restoring the simplicity of the familiar orthogonality relations satisfied by the Jacobi polynomials.

Let

$$
U_{n}=\left(\begin{array}{cc}
u_{-n-1} & v_{-n-1} \\
u_{n} & v_{n}
\end{array}\right)
$$

Since $\left\{u_{n}(z), v_{n}(z)\right\}$ and $\left\{q_{n}(z), p_{n}(z)\right\}$ both provide a basis of eigenfunctions for the associated Jacobi matrix, there is a $2 \times 2$ matrix $S$ ( $z$ dependent, but $n$ independent) such that

$$
P_{n}=U_{n} S,
$$

with $P_{n}$ as in (3.4). Since $P_{0}=I$, we have that

$$
S=U_{0}^{-1} \text {. }
$$

From (2.13), (2.17), (2.18), and (3.40), one checks easily that

$$
u_{0}=G_{1}, \quad v_{0}=z^{1-c} G_{2}, \quad u_{-1}=-\frac{1}{\gamma} G_{3}, \quad v_{-1}=-\frac{\nu}{\gamma} z^{1-c} G_{4},
$$


with $\nu$ and $\gamma$ as in (3.30) and (3.31), and thus

$$
S=\frac{1}{\left(\nu G_{1} G_{4}-G_{2} G_{3}\right)}\left(\begin{array}{cc}
\gamma G_{2} & \nu G_{4} \\
-\gamma z^{c-1} G_{1} & -z^{c-1} G_{3}
\end{array}\right) .
$$

By (4.2), putting $z=x$ to be real in the interval [0,1], we get

$$
P_{m}(x) d \Sigma(x) P_{n}(x)^{T}=U_{m}(x) S(x) d \Sigma(x) S(x)^{T} U_{n}(x)^{T} .
$$

Using (3.42) and (4.5), one computes that

$$
S(x) d \Sigma(x) S(x)^{T}=\left(\begin{array}{cc}
1 & 0 \\
0 & -\mu K^{2}
\end{array}\right) \gamma L \rho(x) d x,
$$

with $K, \gamma, \mu, \rho(x)$ and $L$ as in (3.29), (3.31), (3.33), (3.43), and (3.44).

Let us define

$$
\tilde{u}_{n}(x)=\sqrt{\gamma L} u_{n}(x) \text { and } \tilde{v}_{n}(x)=\sqrt{-\mu K^{2} \gamma L} v_{n}(x) .
$$

Remembering that the set of parameters $a, b$, and $c$ is equivalent to the set of parameters $\alpha, \beta$, and $t$ as defined in (2.9), by using some of the standard functional equations satisfied by the gamma function, one computes easily from (3.29), (3.31), (3.33), and (3.44) that

$$
\begin{gathered}
\gamma L=\frac{\Gamma(-\beta) \Gamma(-2 t-\alpha-\beta) \Gamma(2 t+\alpha+\beta+2)}{\Gamma(-t-\beta) \Gamma(-t-\alpha-\beta) \Gamma(t+1) \Gamma(t+\alpha+1) \Gamma(\beta+1)}, \\
\mu=\frac{\sin (\pi t) \sin \pi(t+\alpha)}{\sin \pi(t+\alpha+\beta) \sin \pi(t+\beta)}
\end{gathered}
$$

and

$$
K=\frac{\Gamma(-t-\alpha-\beta) \Gamma(t+1) \Gamma(\beta) \sin \pi(t+\alpha+\beta)}{\Gamma(-t-\alpha) \Gamma(t+\beta+1) \Gamma(-\beta) \sin \pi(t+\alpha)} .
$$

It is not hard to check that the conditions discussed in Section 2, which ensure the positivity of all $a_{n}$, force the constants $\gamma L$ and $-\mu K^{2} \gamma L$ in (4.8) to be positive. The next theorem shows that, in the basis provided by the functions $\tilde{u}_{n}$ and $\tilde{v}_{n}, n \in \mathbb{Z}$, the simplicity of the orthogonality relations is indeed restored.

Theorem 2. Let $\tilde{u}_{n}$ and $\tilde{v}_{n}$ be defined as in (4.8), then the following orthogonality relations hold

$$
\int_{0}^{1}\left(\tilde{u}_{m}(x) \tilde{u}_{n}(x)+\tilde{v}_{m}(x) \tilde{v}_{n}(x)\right) \rho(x) d x=\delta_{m n}, \text { for all } m, n \in \mathbb{Z},
$$

with

$$
\rho(x)=x^{\beta}(1-x)^{\alpha} .
$$

Proof. From (4.1), (4.7), and (4.8), we compute that

$$
\begin{aligned}
& U_{m}(x) S(x) d \Sigma(x) S(x)^{T} U_{n}(x)^{T} \\
& =\left(\begin{array}{cc}
\tilde{u}_{-m-1} \tilde{u}_{-n-1}+\tilde{v}_{-m-1} \tilde{v}_{-n-1} & \tilde{u}_{-m-1} \tilde{u}_{n}+\tilde{v}_{-m-1} \tilde{v}_{n} \\
\tilde{u}_{m} \tilde{u}_{-n-1}+\tilde{v}_{m} \tilde{v}_{-n-1} & \tilde{u}_{m} \tilde{u}_{n}+\tilde{v}_{m} \tilde{v}_{n}
\end{array}\right) \rho(x) d x,
\end{aligned}
$$

$m, n=0,1,2, \ldots$, with $\rho(x)$ as in (3.43) which, using (2.9), agrees with (4.13). The result follows then from (4.6) and the orthogonality relations (3.10). This establishes the theorem.

We like to finish with a few comments and remarks: 
Remark 1. Observe that, when $t=0$, the functions $u_{n}$ as defined in (2.17) become polynomials. Since, by (2.11), they satisfy the standard three-term recursion relation of the Jacobi polynomials (written in the symmetric form (3.1)), it gives them no other choice than to coincide with the Jacobi polynomials. Also, from (4.10), $\mu=0$ when $t=0$, and thus, remembering the definition of $\tilde{u}_{n}$ and $\tilde{v}_{n}$ in (4.8), the orthogonality relations (4.12) reduce to the standard orthogonality relations satisfied by the Jacobi polynomials (shifted to the interval $[0,1]$ and appropriately normalized to become orthonormal):

$$
\int_{0}^{1} \tilde{u}_{m}(x) \tilde{u}_{n}(x) x^{\beta}(1-x)^{\alpha} d x=\delta_{m n}, \quad m, n=0,1,2, \ldots
$$

Remark 2. The proof of the orthogonality relations (4.12) that we have given above relies on the general spectral theory of self-adjoint second-order difference operators, which guarantees that the orthogonality relations (3.10) hold (see [4]). However, the fact that the spectral matrix can be put into diagonal form as in (4.7), in some appropriate basis, doesn't seem to be ensured by the general theory. Here we would just like to mention that the bispectral property of the associated Jacobi matrix was a strong indication for us that it should be possible to bring the spectral matrix into diagonal form in a basis of bispectral functions. In fact, using the bispectral property (2.7), it is possible (and very instructive) to give an "elementary" proof that the orthogonality relations (4.12) hold for $m \neq n$. For this one writes the operator $B$ in (2.8) in self-adjoint form

$$
\begin{gathered}
B=\frac{1}{\rho(x)} \frac{d}{d x}\left(p(x) \frac{d}{d x}\right), \text { with } \\
p(x)=x^{\beta+1}(1-x)^{\alpha+1} \text { and } \rho(x)=x^{\beta}(1-x)^{\alpha} .
\end{gathered}
$$

By the standard integration by parts argument and using $B \tilde{u}_{n}=\lambda_{n} \tilde{u}_{n}$ and $B \tilde{v}_{n}=$ $\lambda_{n} \tilde{v}_{n}$, this gives that

$$
\left(\lambda_{m}-\lambda_{n}\right) \int_{0}^{1}\left(\tilde{u}_{m} \tilde{u}_{n}+\tilde{v}_{m} \tilde{v}_{n}\right) \rho(x) d x=\left.p(x)\left(W\left(\tilde{u}_{n}, \tilde{u}_{m}\right)+W\left(\tilde{v}_{n}, \tilde{v}_{m}\right)\right)\right|_{0} ^{1}
$$

with $W$ denoting the Wronskian. By a careful analysis, one can show that the sum of the two Wronskians above vanishes both at $x=0$ and $x=1$, only under the conditions that $-1<\alpha<1$ and $-1<\beta<1$. Notice that, as explained in Section 2 , these conditions are precisely those which ensure that there exists some values of the parameter $t$ for which the $a_{n}$ 's (as defined in (2.10)) are positive, so that the associated Jacobi matrix is self-adjoint. This computation gives strong evidence (though not a rigorous proof) that, in the self-adjoint case, the spectral (matrix) measure of the associated Jacobi matrix has only an absolutely continuous part and no discrete masses.

Remark 3. The expression (4.12) makes it natural to consider the functions $\psi_{n}(\theta)$ given by

$$
\frac{1}{\sqrt{2}} \cos ^{\beta+1 / 2}(\theta / 2) \sin ^{\alpha+1 / 2}(\theta / 2)\left(\tilde{u}_{n}\left(\cos ^{2}(\theta / 2)\right)+i \tilde{v}_{n}\left(\cos ^{2}(\theta / 2)\right)\right)
$$

and to express the orthogonality in terms of the integrals

$$
\int_{0}^{2 \pi} \psi_{n}(\theta) \overline{\psi_{m}(\theta)} d \theta=\delta_{n m}
$$


This corresponds to the spectral theorem for $L$ and reduces to the usual Fourier expansion for $\beta=1 / 2, \alpha=-1 / 2$ in which case $\psi_{n}(\theta)=i e^{i n \theta} / \sqrt{2 \pi}$.

Acknowledgements. The first author was supported in part by NSF Grant \#DMS94-00097 and by AFOSR under Contract FDF49620-96-1-0127. The second author is a Research Associate of the Belgian National Fund for Scientific Research.

\section{References}

1. N. I. Akhiezer, The Classical Moment Problem, Hafner Publishing Company, New York, 1965.

2. R. Askey and M. E. H. Ismail, Recurrence Relations, Continued Fractions and Orthogonal Polynomials, Mem. Amer. Math. Soc. 300, 1984.

3. R. Askey and J. Wimp, Associated Laguerre and Hermite polynomials, Proc. Roy. Soc. Edinburgh Sect. A 96 (1984) 15-37.

4. Ju. M. Berezanskii, Expansions in Eigenfunctions of Selfadjoint Operators, Transl. Math. Monographs 17, Amer. Math. Soc., Providence, 1968.

5. S. Bochner, Über Sturm-Liouvillesche Polynomsysteme, Math. Z. 29 (1929), 730-736.

6. P. Barrucand and D. Dickinson, On the associated Legendre polynomials. In: Orthogonal Expansions and Their Continual Analogues (D.T. Haimo, ed.), Southern Illinois U. Press, Edwardsville, 1968, pp.43-50.

7. T. S. Chihara, An Introduction to Orthogonal Polynomials, Gordon and Breach, New YorkLondon-Paris, 1978.

8. Y. Chen and M. E. H. Ismail, Ladder operators and differential equations for orthogonal polynomials, J. Phys. A. 30 (1997), 7818-7829.

9. J. J. Duistermaat and F. A. Grünbaum, Differential equations in the spectral parameter, Commun. Math. Phys. 103 (1986), 177-240.

10. A. Erdélyi, W. Magnus, F. Oberhettinger, and F. G. Tricomi, Higher Transcendental Functions, vol.1 and 2, McGraw-Hill, New York, 1953.

11. F. A. Grünbaum and L. Haine, A theorem of Bochner, revisited. In: Algebraic Aspects of Integrable Systems (A.S. Fokas and I.M.Gelfand, eds.), Progr. Nonlinear Differential Equations Appl., vol. 26, Birkhäuser, Boston, 1997, pp.143-172.

12. Some functions that generalize the Askey-Wilson polynomials, Commun. Math. Phys. 184 (1997), 173-202.

13. L. Haine, Beyond the classical orthogonal polynomials. In: The Bispectral Problem, Montréal 1997, CRM Proc. Lecture Notes, (J. Harnad and A. Kasman, eds.), vol. 14, Amer. Math. Soc., Providence, 1998, pp.47-65.

14. J. Harnad and A. Kasman, Eds. The Bispectral Problem, Montréal 1997, CRM Proc. Lecture Notes, vol. 14, Amer. Math. Soc., Providence, 1998.

15. E. Hellinger, Zur Stieltjesschen Kettenbruchtheorie, Math. Ann. 86 (1922), 18-29.

16. M. E. H. Ismail, J. Letessier, G. Valent, and J. Wimp, Associated Wilson polynomials, Canad. J. Math. 42 (1990), 659-695.

17. M. E. H. Ismail and M. Rahman, The associated Askey-Wilson polynomials, Trans. Amer. Math. Soc. 328 (1991), 201-237.

18. D. R. Masson and J. Repka, Spectral theory of Jacobi matrices in $\ell^{2}(\mathbb{Z})$ and the su $(1,1)$ Lie algebra, SIAM J. Math. Anal. 22 (1991), 1131-1146.

19. G. Palama, Polinomi piú generali di altri classici e dei loro associati e relazioni tra essi funzioni di seconda specie, Riv. Mat. Univ. Parma 4 (1953), 363-383.

20. H. Weyl, Über gewöhnliche Differentialgleichungen mit Singularitäten und die zugehörigen Entwicklungen willkürlicher Funktionen, Math. Ann. 68 (1910), 220-269.

21. J. Wimp, Explicit formulas for the associated Jacobi polynomials and some applications, Canad. J. Math. 39 (1987), 983-1000.

Department of Mathematics, University of California, Berkeley, CA 94720

Department of Mathematics, Université Catholique de Louvain, 1348 Louvain-la-Neuve, BELGIUM 